\title{
Confecção de um modelo experimental in vitro do espaço subaracnóide eqüino para teste de opióides hiperbáricos
}

\author{
Making of an in vitro experimental model of equine subarachnoid space to test hyperbaric opioids
}

\author{
Alexandre da Silva Polydoro ${ }^{\text {I }}$ Alceu Gaspar Raiser ${ }^{\text {II }}$ Cláudio Corrêa Natalini ${ }^{I I I}$ \\ Renata Lehn Linardi ${ }^{\mathrm{IV}}$
}

\section{RESUMO}

Com o objetivo de avaliar as características de distribuição de agentes hiperbáricos no líquido cérebroespinhal (LCE) eqüino, é apresentado um modelo in vitro do espaço subaracnóide confeccionado em policloreto de vinila (PVC) transparente, no qual se adaptou um cateter para a infusão contínua de glicose a 10\%, morfina hiperbárica, buprenorfina hiperbárica e metadona hiperbárica marcadas com azul de metileno para permitir a visualização da distribuição destas substâncias no LCE. Avaliaram-se a distância alcançada pelos diferentes agentes minuto a minuto e a forma de distribuição dos mesmos. Todas as substâncias testadas mostraram comportamento de distribuição vertical a partir da extremidade do cateter, quando se deu o início da migração cranial no LCE, sendo que o avanço máximo registrado foi aos 15 minutos após o término da infusão, com migração de $15 \mathrm{~cm}$ para glicose a $10 \%, 13 \mathrm{~cm}$ para a morfina hiperbárica, $18 \mathrm{~cm}$ para a buprenorfina hiperbárica e $17 \mathrm{~cm}$ para a metadona hiperbárica. O modelo proposto mostrou-se eficiente para a avaliação do comportamento físico dos agentes hiperbáricos no interior do espaço subaracnóide, oferecendo a possibilidade de teste de substâncias a serem utilizadas pela via subaracnóide de cavalos.

Palavras-chave: modelo experimental, subaracnóide, anestesia, hiperbárico.

\section{ABSTRACT}

The aim of this study was to evaluate the distribution characteristics of hyperbaric solutions in equine cerebrospinal fluid (CSF), in an in vitro model of the subarachnoid space. The model was made with transparent PVC in which a catheter was adapted to infuse $10 \%$ glucose, $10 \%$ hyperbaric morphine, hyperbaric buprenorphine, and hyperbaric methadone, dyed with methilene blue in order to visualize the solutions distribution in the CSF. The distance in $\mathrm{cm}$ reached by the different agents every minute as well as the pattern of distribution were evaluated. All tested solutions distributed itself in a forward and vertically pattern from the tip of the catheter. Maximum distance reached after 15 minutes were $15 \mathrm{~cm}$ for $10 \%$ glucose, $13 \mathrm{~cm}$ for hyperbaric morphine, $18 \mathrm{~cm}$ for hyperbaric buprenorphine, and $17 \mathrm{~cm}$ for hyperbaric methadone. The proposed model was efficient to evaluate the distribution pattern of hyperbaric solutions in equine CSF, offering a new possibility to physically test the administration of solutions in the equine subarachnoid space.

Key words: experimental model, subarachnoid, in vitro, hyperbaric.

\section{INTRODUÇÃO}

Poucos são os relatos da utilização de modelos in vitro para investigações científicas envolvendo fármacos anestésicos e/ou analgésicos pela via espinhal.

Para ROBINSON et al. (1994), a distribuição da substância analgésica no interior do espaço subaracnóide é um dos fatores determinantes para o sucesso da anestesia ou analgesia espinhal, sendo que modelos in vitro podem ser utilizados para a investigação dos fatores que afetam a distribuição do anestésico no líquido cérebro-espinhal, podendo servir de base para a posterior utilização clínica. Estes autores desenvolveram um modelo espinhal plástico simulando o espaço subaracnóide, a medula espinhal e a cauda eqüina da espécie humana. O modelo preenchido por Ringer com lactato de sódio com gravidade específica de $1005 \mathrm{~g} \mathrm{~mL}^{-1}$, foi submetido a sucessivas injeções de

'Laboratório de Cirurgia Experimental (LACE), Programa de Pós-graduação em Medicina Veterinária (PPGMV), Universidade Federal de Santa Maria (UFSM), Santa Maria, RS, Brasil. Endereço para correspondência: Rua Ângelo Possebon, 105 apto 403 , Centro, 92310-140, Canoas, RS, Brasil. E-mail: a.polydoro@terra.com.br. *Autor para correspondência.

IIDepartamento de Clínica de Pequenos Animais, UFSM, Santa Maria, RS, Brasil.

IIIInstituto de Ciências Básicas da Saúde (ICBS), Universidade Federal do Rio Grande do Sul (UFRGS), Porto Alegre, RS, Brasil.

${ }^{\mathrm{IV}}$ Department of Veterinary Clinical Sciences, Louisisna State University, Baton Rouge/LA, 70803, USA. 
anestésico local hiperbárico corado com azul de ftalocianina, processadas digitalmente para avaliação. McELLISTREM et al. (1993) relataram a utilização de um modelo in vitro para a determinação da permeabilidade da dura-máter de seres humanos à opióides e anestésicos locais, através da confecção de um aparato constituído de duas câmaras de vidro separadas por uma porção de dura-máter de $3 \mathrm{~cm}^{2}$ e preenchidas com líquido cérebro-espinhal artificial com a mesma osmolalidade do líquido cérebro-espinhal. A permeabilidade foi testada a partir da administração do agente analgésico na câmara " $\mathrm{A}$ ” e em tempos prédeterminados. Os autores também coletaram amostra na câmara “B”, realizando a cromatografia e a determinação da difusão das substâncias através da dura-máter.

Na espécie humana, relato de cefaléia após punção subaracnóide é uma das complicações de maior ocorrência clínica e sem dúvida é motivo de grande preocupação. Modelos experimentais também já foram utilizados para teste de diferentes agulhas utilizadas nos acessos subaracnóides, proporcionando um conhecimento prévio das possíveis lesões teciduais que possam produzir este tipo de desconforto após a punção (APAN et al., 2002). Em outro estudo in vitro, REINA et al. (2000) realizaram sucessivas punções em segmentos de dura-máter retiradas de seres humanos que realizaram doação de órgãos depois de constatada morte cerebral com o objetivo de determinar o possível dano tecidual produzido por diferentes tipos de agulhas (Whitacre e Quincke). Os autores chegaram a conclusão que o tipo de lesão produzido pelas agulhas testadas é diferenciado pela característica de corte de cada uma.

PARLOW et al. (1999) investigaram a influência da adição de morfina e fentanil na densidade de agentes anestésicos locais hiperbáricos num modelo experimental in vitro do espaço subaracnóide da espécie humana, em que observaram a direção e a extensão de tais substâncias marcadas com azul de metileno.

Em Medicina Veterinária, não há relatos da utilização de modelos espinhais in vitro em nenhuma espécie servindo como base para determinação de características físicas de substâncias analgésicas administradas pela via epidural ou subaracnóide, e sendo assim, este estudo objetivou relatar a confecção e avaliar a eficácia de um modelo experimental in vitro do espaço subaracnóide na avaliação da distribuição de opióides hiperbáricos a serem utilizados posteriormente in vivo.

\section{MATERIAL E MÉTODO}

O trabalho foi desenvolvido junto ao Laboratório de Pesquisa em Eqüinos da Louisiana State
University, School of Veterinary Medicine, Baton Rouge - EUA, sendo devidamente aprovado pelo comitê de ética em pesquisa da referida universidade.

Desenvolveu-se um modelo experimental do espaço subaracnóide de um cavalo adulto, utilizandose uma coluna de PVC (policloreto de vinila) transparente ${ }^{\mathrm{a}}$, medindo $165 \mathrm{~cm}$ de comprimento por $2,5 \mathrm{~cm}$ de diâmetro, montada $115 \mathrm{~cm}$ na posição horizontal e $50 \mathrm{~cm}$ na posição vertical em ângulo de $130^{\circ}$, com capacidade para albergar $600 \mathrm{~mL}$ de líquido cérebro-espinhal eqüino (LCE). O dispositivo foi graduado com uma escala em centímetros estendendose $150 \mathrm{~cm}$ a partir do ponto onde foi adaptado um cateter subaracnóide $19 \mathrm{G}$, colocado a $10 \mathrm{~cm}$ da extremidade horizontal do modelo, por onde foram infundidas as soluções hiperbáricas testadas. Um segmento de silicone com 1,2cm de diâmetro foi adaptado no interior da coluna de PVC mimetizando a medula espinhal, sendo realizado posteriormente o fechamento vedado da extremidade horizontal do modelo (Figura 1a). O líquido cérebro-espinhal para o preenchimento dos modelos foi obtido a partir de eqüinos sacrificados para fins de pesquisa no setor de Patologia da Louisiana State University, sendo o mesmo coletado através da punção da cisterna magna com agulha 200x12 logo após o sacrifício dos animais e acondicionado em bolsas plásticas destinadas para o armazenamento de plasma e mantido refrigerado a $4^{\circ} \mathrm{C}$ em um período máximo de 48h antes da experimentação. Antes do preenchimento do modelo, o LCE foi aquecido em banho-maria a $37^{\circ} \mathrm{C}$. Seqüencialmente preencheu-se o modelo com líquido cérebro-espinhal eqüino previamente aquecido, quando se realizava a retirada de uma amostra do LCE do interior do modelo para a obtenção do valor da densidade do mesmo, determinada por refratometria. A temperatura do modelo foi mantida constante durante a experimentação através de uma manta com água circulante aquecida colocada sob o modelo experimental fixado em bancada (Figura 1b).

Para a obtenção de substâncias hiperbáricas a serem infundidas no modelo, diluiu-se morfinac, buprenorfina $^{\mathrm{d}}$ e metadona ${ }^{\mathrm{e}}$ em igual volume com glicose $^{\mathrm{f}}$ a 5\% e 10\%. Após a adição de glicose a 5\% e a $10 \%$, tais agentes hiperbáricos foram marcados com azul de metileno $(0,1 \mathrm{~mL}$ da solução pura de azul de metileno em $5 \mathrm{~mL}$ de opióide hiperbárico) e tiveram a densidade de cada solução determinada por refratometria. As misturas com densidade maior ou igual a 1030 foram infundidas no modelo experimental para avaliação do comportamento físico no líquido cérebro espinhal, em relação à difusão e à distância percorrida.

O teste dos opióides hiperbáricos constou da infusão contínua de $5 \mathrm{~mL}$ em 5 minutos de cada agente hiperbárico por bomba de infusão à seringag , sendo que ao término da infusão no modelo e a cada minuto subseqüente, registrou-se o avanço alcançado 


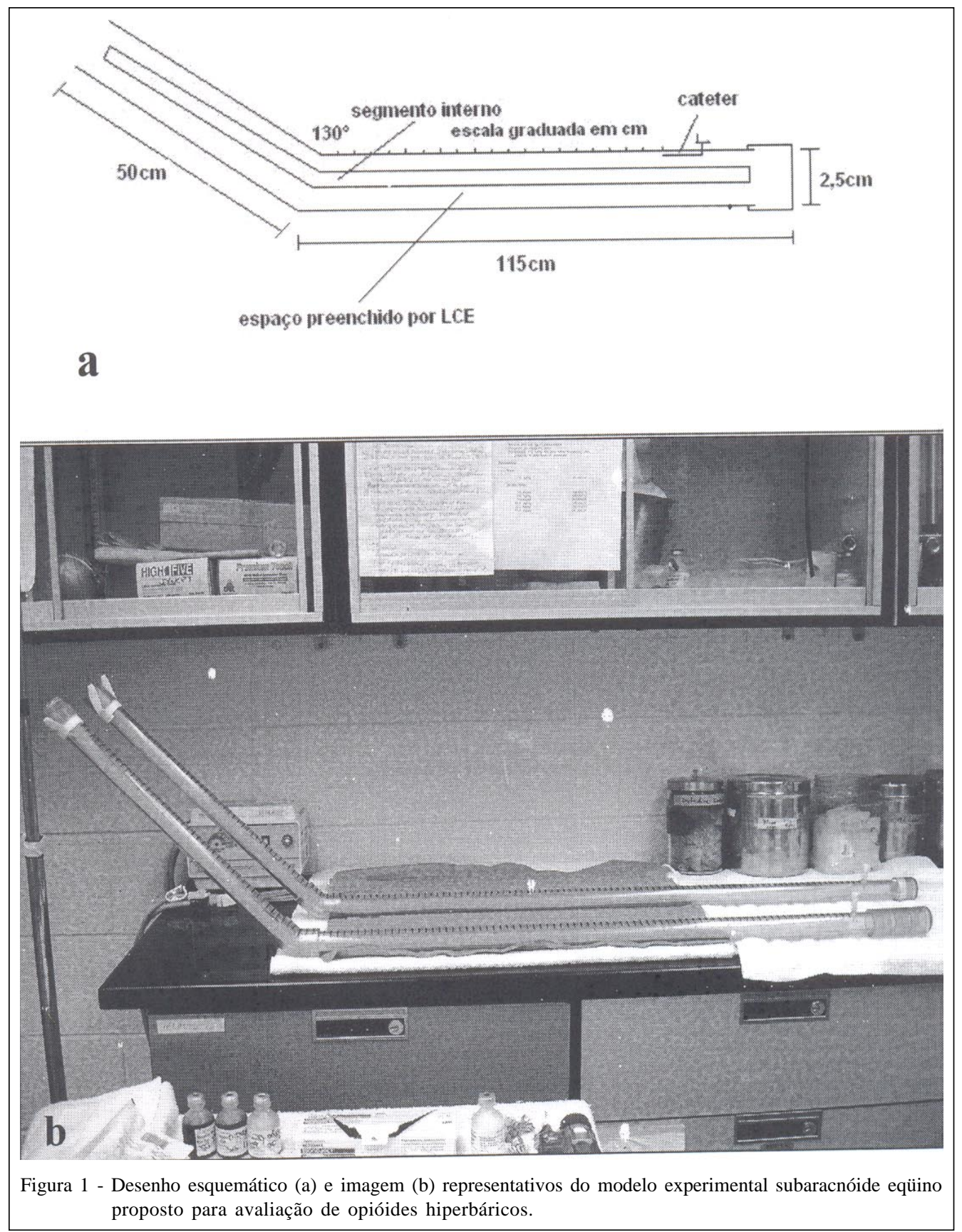

pelo fármaco hiperbárico, até haver a parada da migração das substâncias no modelo. Para cada agente testado, o modelo foi drenado e preenchido com novo líquido cérebro-espinhal.

\section{RESULTADOS E DISCUSSÃO}

LUI et al. (1998) descreveram em uma pesquisa na qual se investigou a densidade do líquido cérebro-espinhal (LCE) e de soluções anestésicas espinhais que, em termos de baricidade, substâncias com densidade superior a 1030 mostram diferença significativa em relação à densidade do LCE. No presente estudo, a determinação da densidade do líquido cérebro-espinhal eqüino depois de preenchido o modelo foi de $1006 \pm 1$,1, sendo que soluções com gravidade específica superior a este valor foram consideradas hiperbáricas em relação ao LCE. A determinação da gravidade específica de cada agente infundido no modelo foi de 1043 para glicose a $10 \%$, 1042 para morfina + glicose $10 \%, 1038$ para metadona + glicose $10 \%$ e 1034 para buprenorfina + glicose a 10\%. 
Os fármacos opióides associados com glicose a $5 \%$ não mostraram valores de densidade mínimos considerados aptos para participarem do estudo.

O modelo proposto neste estudo, preenchido com líquido cérebro-espinhal de cavalos, foi efetivo na avaliação do comportamento físico das substâncias estudadas conforme metodologia proposta, permitindo desta forma servir de base para uma prévia proposição do efeito dos agentes in vivo. ROBINSON et al. (1994) relatam que modelos experimentais podem ser utilizados para investigação de fatores de distribuição de diversas substâncias, pois a distribuição do fármaco injetado no espaço subaracnóide é um fator determinante para o sucesso da técnica. Com isso pode-se estimar o comportamento físico de distribuição previamente ao uso clínico, minimizando-se o risco de efeitos indesejáveis, aumentando o sucesso da técnica proposta.

O comportamento dos fármacos opióides hiperbáricos marcados com azul de metileno durante a administração por infusão contínua a uma taxa de $1 \mathrm{~mL}$ $\mathrm{min}^{-1}$, por bomba à seringa no modelo, mostrou que a morfina a $10 \%$, a buprenorfina a $10 \%$ e a metadona a $10 \%$ apresentaram movimento vertical no líquido cérebro-espinhal no ponto de injeção (extremidade do cateter subaracnóide), fazendo contato direto com o segmento de silicone interno do modelo que mimetizou a medula fazendo uma espécie de depósito no assoalho do modelo, quando houve o avanço do agente opióide hiperbárico na direção do segmento angulado do modelo (Figura 2). PARLOW et al. (1999), em um estudo in vitro em que utilizaram um modelo de PVC transparente do espaço subaracnóide de um indivíduo da espécie humana, comentam que a injeção lenta das substâncias a serem estudadas deve ser lenta o suficiente para minimizar ou anular o efeito de turbulência. No modelo aqui testado, não foi observado tal efeito, sendo que a velocidade de administração dos agentes foi a mesma utilizada pelos autores supracitados.

O registro do avanço em centímetros da glicose a $10 \%$ e dos agentes opióides hiperbáricos a $10 \%$ no modelo foi realizado até os 15 minutos após o término da injeção por infusão por bomba à injeção, tempo este em que houve progressão das substâncias, até uma distância máxima de $15 \mathrm{~cm}$ para a glicose a 10\%, $13 \mathrm{~cm}$ para a morfina a $10 \%, 18 \mathrm{~cm}$ para a buprenorfina a $10 \%$, e $17 \mathrm{~cm}$ para a metadona a $10 \%$. A Tabela 1 mostra os resultados do avanço dos agentes testados no modelo in vitro ao término da administração subaracnóide e de minuto a minuto até 15 minutos após a administração. O modelo permitiu a adequada

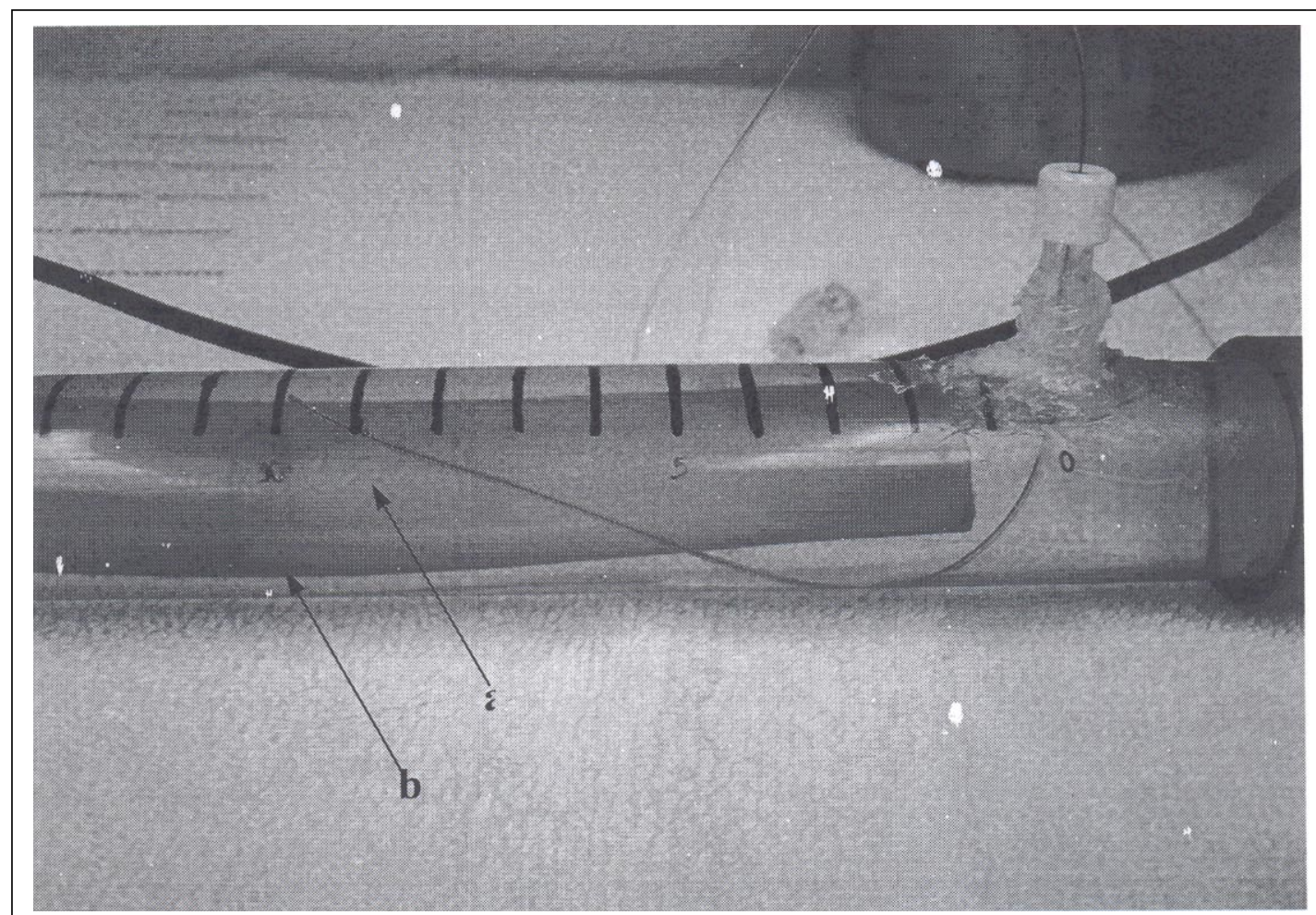

Figura 2 - Imagem do modelo preenchido com LCE mostrando movimento vertical do opióide hiperbárico (a) e depósito no assoalho do modelo (b). 
Tabela 1 - Registro do avanço (em centímetros) dos fármacos testados no modelo experimental in vitro do espaço subaracnóide.

\begin{tabular}{lcccc}
\hline Tempo(min) & $\begin{array}{c}\text { Glicose } \\
10 \%\end{array}$ & $\begin{array}{c}\text { Morfina } \\
10 \%\end{array}$ & $\begin{array}{c}\text { Buprenorfina } \\
10 \%\end{array}$ & $\begin{array}{c}\text { Metadona } \\
10 \%\end{array}$ \\
\hline 0 & 13 & 11 & 15 & 15 \\
1 & 13 & 12 & 16 & 15 \\
2 & 14 & 12 & 17 & 16 \\
3 & 14 & 13 & 17 & 17 \\
4 & 15 & 13 & 17 & 17 \\
5 & 15 & 13 & 17 & 17 \\
6 & 15 & 13 & 18 & 17 \\
7 & 15 & 13 & 18 & 17 \\
8 & 15 & 13 & 18 & 17 \\
9 & 15 & 13 & 18 & 17 \\
10 & 15 & 13 & 18 & 17 \\
11 & 15 & 13 & 18 & 17 \\
12 & 15 & 13 & 18 & 17 \\
13 & 15 & 13 & 18 & 17 \\
14 & 15 & 13 & 18 & 17 \\
15 & 15 & 13 & 18 & 17 \\
\hline
\end{tabular}

visualização das soluções que ficaram evidenciadas pela coloração do azul de metileno.

\section{CONCLUSÕES}

Diante da metodologia proposta neste estudo, pode-se concluir que: o modelo experimental in vitro confeccionado em PVC transparente nas dimensões propostas e preenchido com LCE eqüino, é eficiente para teste de opióides hiperbáricos pela via subaracnóide permite a visualização direta do comportamento das substâncias hiperbáricas nele injetadas, e oferece subsídios para escolha de substâncias a serem administradas in vivo, quando for o caso.

\section{AGRADECIMENTOS}

Os autores agradecem à Louisiana State University - USA pela concessão do Laboratório de Pesquisa em Eqüinos, onde foi realizada a referida pesquisa, que foi parte do trabalho apresentado como requisito parcial para obtenção do título de Doutor em Medicina Veterinária (PPGMV).

\section{FONTESDEAQUISIÇÃO}

${ }^{a}$ Clear rigid PVC tube, Harvel Plastics, Inc., Easton, PA, USA. 'Theracath epidural catheter, Arrow International Inc., Reading, PA, USA.

'Infumorph ${ }^{\circledR}$, Elkin-Sinn, Cherry Hill, NJ, USA.

${ }^{\mathrm{d} B u p r e n e x}$ 0,3mg/mL, Reckitt and Coleman Pharmaceuticals Ltd., Richmond, VA, USA.

${ }^{e}$ Methadone Hydrochloride $10 \mathrm{mg} / \mathrm{mL}$, Alpharma Inc., Lincolnton, NC, USA.

${ }^{\mathrm{f}}$ Dextrose $10 \%$, The Butler Company, Dublin, OH, USA

gMedfusion 2010i, Medex Inc., Cheshire, CT, USA.

\section{REFERÊNCIAS}

ANGLE, P.J. et al. Epidural catheter penetration of human dural tissue. In vitro investigation. Anesthesiology, v.100, n.6, p.1491-1496, 2004.

APAN, A. et al. The effect of changing pressures on dural puncture and leak with various spinal needles on an in vitro model. Journal of Clinical Neuroscience, v.9, n.6, p.677679, 2002.

LUI, A.C.P. et al. Densities of cerebrospinal fluid and spinal anaethetic solutions in surgical patients at body temperature. Canadian Journal of Anaesthesia, v.45, n.4, p.297-303, 1998.

McELLISTREM, R.F. et al. In vitro determination of human dura mater permability to opioids and local anaesthetics. Canadian Journal of Anaesthesia, v.40, n.2, p.165-169, 1993.

PARLOW, J.L. et al. Addition of opioids alters the density and spread of intrathecal local anesthetics? An in vitro study. Canadian Journal of Anesthesia, v.46, n.1, p.66-70, 1999.

REINA, M.A. et al. An in vitro study of dural lesions produced by 25-gauge Quincke and Whitacre needles evaluated by scanning electron microscopy. Regional Anesthesia and Pain Medical, v.25, n.4, p.393-402, 2000.

ROBINSON, R.A. et al. In vitro modeling of spinal anesthesia. A digital video image processing technique and its application to catheter characterization. Anesthesiology, v.81, n.4, p.1053-1060, 1994. 